\title{
Association of the hypertriglyceridemic waist phenotype and severity of acute pancreatitis
}

\author{
Yanbing Ding ${ }^{1,2+}$, Min Zhang ${ }^{1,2+}$, Lisheng Wang ${ }^{3 \dagger}$, Tao Yin ${ }^{2}$, Ningzhi Wang ${ }^{1,2}$, Jian $\mathrm{Wu}^{1,2}$, Jiehua Zhi ${ }^{1,2}$, \\ Weiwei Chen ${ }^{4}$, Keyan $\mathrm{Wu}^{1,2}$, Weijuan Gong ${ }^{1,2,5}$, Weiming Xiao ${ }^{1,2}$, Zhenglei Xu ${ }^{3^{*}}$ and Guotao $\mathrm{Lu}^{1,2^{*}}$
}

\begin{abstract}
Background: The aim of this study was to evaluate the effect of a simple visceral obesity phenotype, known as the hypertriglyceridemic waist phenotype and its quantitative indicator waist circumference index on the severity of acute pancreatitis.

Materials and methods: Diagnosis and severity analysis of acute pancreatitis were determined according to the Atlanta classification guidelines, revised in 2012. We considered the hypertriglyceridemic waist phenotype as characterized by increased waist circumference and elevated triglyceride concentrations. We investigated the association between the acute pancreatitis severity and hypertriglyceridemic waist phenotype, including waist circumference index.

Results: The hypertriglyceridemic waist phenotype was significantly associated with systemic inflammatory response syndrome, organ failure, and severe acute pancreatitis. The median waist circumference index and demonstration of hypertriglyceridemic waist phenotype were positively correlated with acute pancreatitis severity. In addition, multivariate logistic analysis showed that patients with the hypertriglyceridemic waist phenotype had 1.664 times the risk of organ failure and 1.891 times the risk of systemic inflammatory response syndrome, compared with the other groups.

Conclusion: Upon admission, the hypertriglyceridemic waist phenotype was strongly associated with acute pancreatitis in patients. This phenotype, including waist circumference index, might be a simple method for evaluating individuals at high risk of severe acute pancreatitis.
\end{abstract}

Keywords: Acute pancreatitis, Hypertriglyceridemic waist phenotype, Waist circumference index, Retrospective study

\section{Introduction}

Acute pancreatitis (AP) is an inflammatory disease of the pancreas and one of the main causes of acute abdominal pain [1]. The incidence of AP is increasing annually and ranges from 4.9 to 80 cases per 100,000 individuals worldwide [2]. AP, especially severe acute pancreatitis (SAP), imposes a serious burden on patients and society. SAP accounts for 10 to $20 \%$ of all AP cases

\footnotetext{
* Correspondence: xzllsx@hotmail.com; gtlu@yzu.edu.cn

${ }^{\dagger}$ Yanbing Ding, Min Zhang and Lisheng Wang are contributed equally to this work

${ }^{3}$ Department of Gastroenterology, The second Clinical Medical College (Shenzhen People's Hospital), Jinan University, Shenzhen 518000, Guangdong, China

'Department of Gastroenterology, Affiliated Hospital of Yangzhou University, Yangzhou University, No. 386 Hanjiang Media Road, Yangzhou 225000, Jiangsu, China

Full list of author information is available at the end of the article
}

and is often accompanied by systemic inflammatory response syndrome (SIRS) and multiple organ dysfunction syndrome, with a mortality rate of up to $30 \%[3,4]$. Early and accurate assessment of AP severity and prognosis is therefore of great significance for clinical diagnosis and treatment.

Recent studies have shown that abnormal lipid metabolism plays an important role in the inflammatory response and prognosis of AP and is related to conditions like obesity and hypertriglyceridemia. Obesity is a state of excessive fat tissue and is a well-known risk factor for AP that can exacerbate inflammation [5-7]. A meta-analysis showed that obesity is associated with local complications, organ failure, and high mortality in patients with AP [8].Further studies found that visceral fat, not peripheral fat, is closely related to the formation

(c) The Author(s). 2019 Open Access This article is distributed under the terms of the Creative Commons Attribution 4.0 International License (http://creativecommons.org/licenses/by/4.0/), which permits unrestricted use, distribution, and 
of pseudocysts in patients with AP and SIRS. In contrast, patients with excessive visceral fat are more likely to have SAP [9]. Apart from obesity; hypertriglyceridemia is another key lipid metabolism disease that affects AP $[10,11]$. It has been well recognized that hypertriglyceridemia is not only the cause of AP but also a factor associated with worsened prognosis in patients with AP [12]. Clinical studies have suggested that elevated serum triglycerides (TG) are independently associated with organ failure in patients with AP [13].

The concept of the hypertriglyceridemic waist (HTGW) phenotype was put forward by Lemieux et al., who suggested that this simple phenotype can serve as a useful marker of metabolic abnormalities [14]. The waist circumference (WC) index (WTI) is a quantitative indicator of the HTGW phenotype [15]. Many previous studies have suggested that the HTGW phenotype can be used to identify the characteristics of excessive visceral adipose tissue, ectopic fat, and metabolic syndrome (Mets) [16-18]. Other studies demonstrated that the HTGW phenotype is a risk factor for cardiovascular disease and is closely related to prodromal diabetes and hypertension [19-21]. To the best of our knowledge, there is no research on the association between the HTGW phenotype and AP. The purpose of this study was to evaluate the effects of the HTGW phenotype on disease severity in patients with AP.

\section{Methods}

\section{Inclusion and exclusion criteria}

We retrospectively evaluated patients diagnosed with AP who were admitted to the Affiliated Hospital of Yangzhou University from January 1, 2013, to December 31, 2016. The diagnosis of AP required at least two of the following features: abdominal pain, biochemical evidence of pancreatitis (amylase or lipase elevated $>3$ times above the upper limit of normal), and/or radiographic evidence of pancreatitis on cross-sectional imaging [22]. The cause of AP was considered to be biliary if gallstones or biliary sludge were observed on imaging examinations, including computed tomography (CT), magnetic resonance cholangiopancreatography, and ultrasonography [23, 24]. Hypertriglyceridemic acute pancreatitis (HTG-AP) was characterized by the presence of serum hypertriglyceridemia $(\geq 1000 \mathrm{mg} / \mathrm{dL})$ or by visible lactescent blood with serum hypertriglyceridemia $500-1000 \mathrm{mg} / \mathrm{dL}$ without any other causes [25-27]. We considered patients as exhibiting alcoholic AP if they consumed $>50 \mathrm{~g}$ per day for $>5$ years, or if the patient consumed excessive alcohol shortly before the onset of AP and other causes were ruled out [28-30]. The exclusion criteria were age $>70$ or $<18$ years, recurrent pancreatitis, malignant tumor, ascites, pregnancy, and incomplete information. Because of the retrospective nature of this study, informed consent was waived, and the study was approved by the Ethics Committee of our hospital.

\section{Clinical measurements}

Once admitted, each patient's height was measured while standing with normally aligned shoulders and without shoes. The measurement was read to $0.1 \mathrm{~cm}$. Body mass index (BMI) was calculated as the weight (kg) divided by height $\left(\mathrm{m} / \mathrm{m}^{2}\right)$ squared. Abdominal CT images were used to estimate WC. We selected the navel level on abdominal $\mathrm{CT}$, and the abdominal cavity was considered an ellipse. Then the horizontal axis and vertical axis of the navel level were measured. At last, the WC (defined as the perimeter) was measured using the standard ellipse formula, as shown in Additional file 1 :Figure S1.

\section{Definitions of HTGW phenotype}

Subjects were categorized among four phenotypes based on pre-determined cutoff points [14]. The normal waist normal TG (NWNT) group exhibited $\mathrm{WC}<90 \mathrm{~cm}$ for men and $<80 \mathrm{~cm}$ for women, and serum TG concentrations $<1.7 \mathrm{mmol} / \mathrm{L}$. The normal waist hypertriglyceridemia (NWET) group exhibited $\mathrm{WC}<90 \mathrm{~cm}$ for men, $<80 \mathrm{~cm}$ for women, and serum TG concentrations $\geq 1.7$ $\mathrm{mmol} / \mathrm{L})$. The enlarged WC normal TG (EWNT) group exhibited $W C \geq 90 \mathrm{~cm}$ for men, $\geq 80 \mathrm{~cm}$ for women, and serum TG concentrations $<1.7 \mathrm{mmol} / \mathrm{L}$. Finally, the HTGW group exhibited WC $\geq 90 \mathrm{~cm}$ for men and $\geq 80$ $\mathrm{cm}$ for women, and serum TG concentrations $\geq 1.7$ $\mathrm{mmol} / \mathrm{L}$. The WTI formula was as follows: WTI $\left(\mathrm{cm}^{*} \mathrm{mmol} / \mathrm{L}\right)=\mathrm{WC}(\mathrm{cm}) \times \mathrm{TG}(\mathrm{mmol} / \mathrm{L})[15]$.

\section{The severity of acute pancreatitis}

AP was categorized as mild (MAP), moderately severe (MSAP), and SAP, according to the 2012 revised Atlanta classification [28]. MSAP was associated with transient organ failure and/or local or systemic complications within $48 \mathrm{~h}$. Patients with SAP exhibited persistent organ failure, involving one or more organs and lasting $>48 \mathrm{~h}$.

\section{Systemic inflammatory response syndrome}

SIRS was defined as the existence of two or more of the following [28]: (1) temperature $>38^{\circ} \mathrm{C}$ or $<36^{\circ} \mathrm{C}$; (2) heart rate $>90$ beats $/ \mathrm{min}$ or hypotension (systolic blood pressure $<90 \mathrm{mmHg}$ or $>40 \mathrm{mmHg}$ lower than baseline); (3) shortness of breath $(>20 / \mathrm{min})$ or hyperventilation $\left(\mathrm{PaCO}_{2}<32 \mathrm{mmHg}\right)$; and (4) peripheral blood leukocyte count $>12 \times 10^{9} / \mathrm{L}$, or neutral rod-shaped granulocyte ratio $>10 \%$. However, other factors that could potentially cause these changes were systematically excluded. 


\section{Organ failure}

To determine organ failure, we evaluated the respiratory, circulatory, and renal systems. Organ failure was determined if the score was $\geq 2$ points according to the revised Atlanta Classification Recommendation Marshall score system. [28]

\section{Statistical analyses}

Statistical analyses were performed using SPSS. Descriptive statistics were used to analyze the baseline characteristics of the study population. We performed between-group comparisons of the characteristics and variables among the various severity groups. Normally distributed, continuous variables were expressed as mean \pm standard deviation, and between-group comparisons were performed using an independent sample t-test; one-way ANOVA was performed when three groups were compared.

Non-normally distributed variables were expressed as medians (interquartile range) and compared using the non-parametric rank sum test. We expressed categorical data as numbers and scales, and the Kruskal-Wallis test or chi-square test was used for between-group comparisons. Categorical data were compared using the Mann-Whitney U test or chi-square test. The following indicators were selected: age, gender, BMI, hypertension, diabetes, coronary heart disease, smoking, and alcohol consumption. The HTGW phenotype was the independent variable, whereas organ failure was the dependent variable during multivariable regression analyses.

\section{Results}

Cohort baseline characteristics

From January 2013 to December 2016, 991 patients were diagnosed with AP. Of these, 510 satisfied the inclusion criteria, as shown in the flowchart (Fig. 1). The patients' demographic and clinical characteristics are shown in Table 1. The mean patient age was $47.7 \pm 12.0$ years, and $62.6 \%$ were male. Smokers accounted for 25.1, and $16.9 \%$ of the population reportedly consumed alcohol. Other conditions reported by patients were hypertension (7.5\%), diabetes $(15.9 \%)$, and coronary heart disease (2.4\%).

Patients were assigned to one of three groups according to their WC and TG data. There were 131 patients in the NWNT group, 194 in the EWNT/NWET groups, and 185 in the HTGW group. The mean age for the groups was $53.7 \pm 11.7$ years (NWNT), $49.7 \pm 11.4$ years (EWNT/NWET), and $42.5 \pm 10.2$ years (HTGW). As expected, BMI, TG, WC, and WTI were all significantly higher in the HTGW group than in the NWNT and EWNT/NWET groups. There were no significant differences in alcohol consumption and smoking behaviors among the three groups. In terms of underlying disease, significantly more patients in the HTGW group exhibited diabetes, as shown in Table 1.

The causes of AP were biliary in 144 (28.2\%), alcoholic in 34 (6.7\%), hypertriglyceridemic in 205 (40.2\%), and unknown in 127 (24.9\%). As shown in Table 1, biliary issues were the leading cause of AP (51.1\%) in the NWNT group. In contrast, the leading cause of AP in the HTGW group was hypertriglyceridemia, accounting for

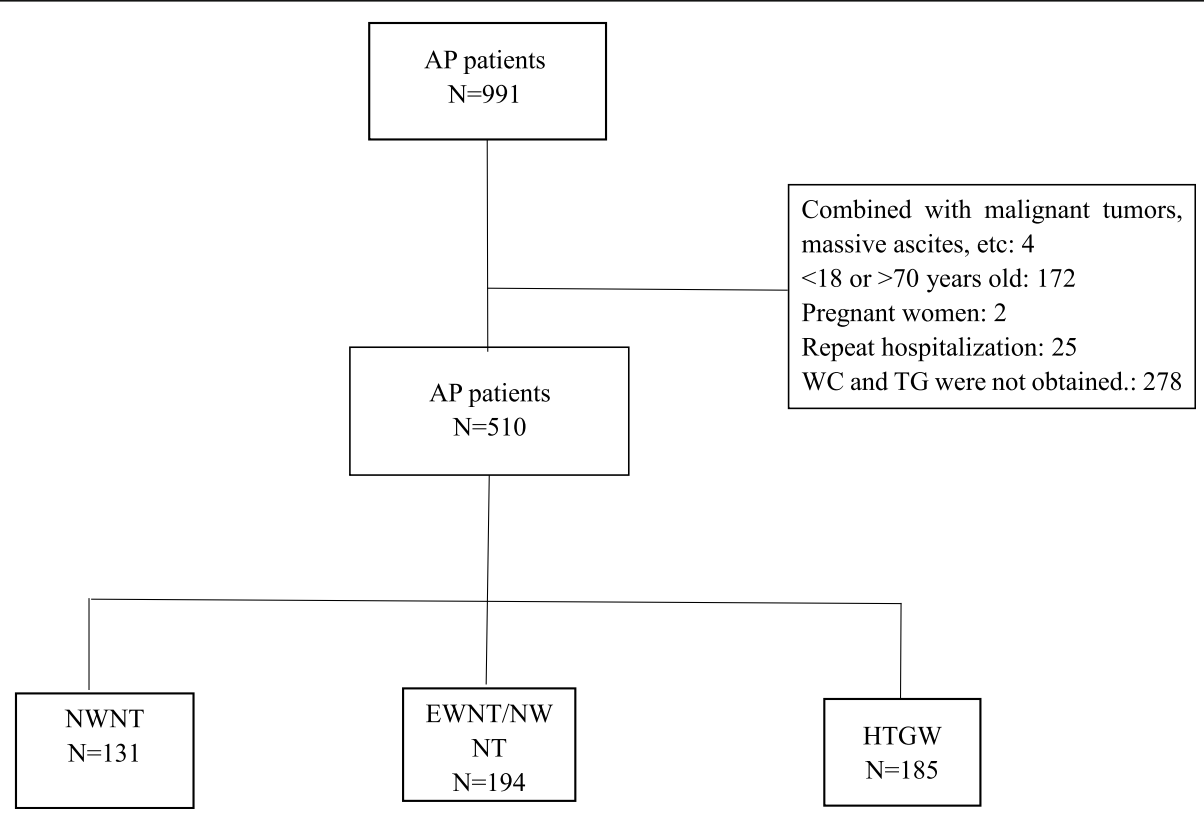

Fig. 1 The distribution of hypertriglyceridemic waist phenotype 
Table 1 The cohort baseline characteristics

\begin{tabular}{|c|c|c|c|c|c|}
\hline Viables & Cohort $N=510$ & NWNT $n=131$ & EWNT/NWET $n=194$ & HTGW $n=185$ & $P$ value $^{a}$ \\
\hline Age, years (mean $\pm S D)$ & $47.9 \pm 11.9$ & $53.7 \pm 11.7^{\#}$ & $49.7 \pm 11.4^{8}$ & $42.5 \pm 10.2$ & $<0.001$ \\
\hline Male (men \%) & 319 (62.6\%) & $84(64.1 \%)$ & $121(62.4 \%)$ & $114(61.6 \%)$ & 0.901 \\
\hline $\mathrm{BMI} \mathrm{I}^{*}$ & $25.5 \pm 5.1$ & $22.2 \pm 5.4^{\#}$ & $25.3 \pm 4.0^{\&}$ & $27.9 \pm 4.6$ & $<0.001$ \\
\hline WC & $87.0 \pm 9.4$ & $78.3 \pm 6.2^{\#}$ & $85.6 \pm 7.3^{\&}$ & $94.5 \pm 7.2$ & $<0.001$ \\
\hline WTI (IQR) & $221.7(10.7-3712.6)$ & $68.9(17.8-145.2)^{\#}$ & $194.9(10.7-3320.0)^{\&}$ & 713.7(150.8-3712.6) & $<0.001$ \\
\hline Smoking & $128(25.1 \%)$ & $29(23.6 \%)$ & $56(31.3 \%)$ & $43(24.9 \%)$ & 0.246 \\
\hline Alcoholic & $86(16.9 \%)$ & $18(14.6 \%)$ & $36(20.7 \%)$ & $32(18.4 \%)$ & 0.412 \\
\hline Hypertension & $38(7.5 \%)$ & $13(9.9 \%)$ & $15(7.7 \%)$ & $10(5.4 \%)$ & 0.316 \\
\hline Diabetes & 81 (15.9\%) & $6(4.6 \%)^{\#}$ & $30(15.5 \%)^{\&}$ & $45(24.3 \%)$ & $<0.001$ \\
\hline Coronary heart disease & $12(2.4 \%)$ & $4(3.1 \%)$ & $7(3.6 \%)$ & $1(0.5 \%)$ & 0.073 \\
\hline Etiology $\#$ \& \& & & & & & 0.002 \\
\hline Biliary & $144(28.2 \%)$ & $67(51.1 \%)$ & $58(29.9 \%)$ & 19 (10.3\%) & \\
\hline Alcohol & $34(6.7 \%)$ & $9(6.9 \%)$ & $16(8.2 \%)$ & $9(4.9 \%)$ & \\
\hline Hypertriglyceridemia & $205(40.2 \%)$ & $5(3.8 \%)$ & 76 (39.2\%) & $124(67 \%)$ & \\
\hline Others & 127 (24.9\%) & 50 (38.2\%) & 44 (22.7\%) & 33 (17.8\%) & \\
\hline
\end{tabular}

arepresents comparing among the three groups;

"The HTGW compared with the NWNT group, $P<0.05$

${ }^{\&}$ The HTGW compared with the EWNT/NWET group, $P<0.05$

Abbreviations: WC Waist circumference,WTI Waist circumference index,BMI Body Mass Index,

$I Q R$ interquartile range

$67.0 \%$ and conforming to the definition of the HTGW phenotype.

\section{Comparison of the severity among the NWNT, EWNT/ NWET, and HTGW subgroups}

According to the 2012 revised Atlanta Classification system, there were $356(69.8 \%)$ patients with MAP disease, 120 (23.5\%) with MSAP disease, and 34 (6.7\%) with SAP disease. The proportion of MAP decreased across the NWNT (77.1\%), EWNT/NWET (73.2\%), and HTGW subgroups (61.1\%), whereas the proportion of SAP gradually increased from 3.8 to $10.8 \%$. There were significant differences in AP severity among the three groups $(P=0.002)$.

As evidenced by Ranson score, the CTSI score, and the prevalence of SIRS and organ failure, patients with HTGW had more severe disease, as shown in Table 2. Furthermore, in the HTGW group, white blood cells (WBCs), and glucose were higher than the respective values in the other groups, whereas creatinine $(\mathrm{Cr})$, blood urea nitrogen (BUN), and lactate dehydrogenase (LDH) did not significantly differ among the groups (Table 2).

\section{Correlation of the severity of AP with the percentage of HTGW and WTI}

We compared clinical features, including the percentage of HTGW and WTI, across the MAP, MSAP, and SAP subgroups. As shown in Table 3, there were no differences in age, gender, and smoking and drinking behaviors across those with MAP, MSAP, and SAP disease. Upon examination of biochemical indicators, which helped indicate AP prognosis (WBC, LDH, Cr, and BUN), those with SAP had showed significantly higher values than the other two groups, indicating a poorer prognosis. Additionally, our study showed that there were no significant differences in BMI among the groups. However, WC and TG levels were higher in the SAP group than in the other two groups. The proportion of the HTGW phenotype grew progressively larger across the MAP (31.7\%), MSAP (43.3\%), and SAP groups (58.8\%). We investigated WTI to further confirm the relationship between the HTGW phenotype and AP severity. Consistent with the results for the HTGW phenotype, in the SAP group, WTI was significantly higher than that in the MAP and MSAP groups.

\section{Logistic regression analysis of organ failure in patients with AP}

Finally, we investigated whether organ failure correlated with the epidemiology and clinical features. A multivariate logistic regression analysis was performed, and the results showed that patients with the HTGW phenotype had 1.664 times the risk of organ failure $(\mathrm{OR}=1.664,95 \% \mathrm{CI}$ 1.033-2.680, $P=0.036)$ and 1.891 times the risk of SIRS $(\mathrm{OR}=1.891,95 \%$ CI 1.192-2.998, $P=0.007)$ compared with the other groups, as shown in Table 4. It is worth noting that patients with diabetes appear more prone to organ failure $(\mathrm{OR}=1.722$, 95\% CI 1.018-2.915, $P=0.043)$ and SIRS $(\mathrm{OR}=1.848,95 \% \mathrm{CI} 1.083-3.153, P=0.024)$. 
Table 2 Comparison of the severity of the three groups

\begin{tabular}{|c|c|c|c|c|c|}
\hline Viables & Cohort $N=510$ & NWNT $n=131$ & EWNT/NWET $n=194$ & HTGW $n=185$ & $P$ value $^{a}$ \\
\hline Severity of $A P^{\#, \&}$ & & & & & 0.002 \\
\hline MAP & $356(69.8 \%)$ & $101(77.1 \%)$ & $142(73.2 \%)$ & $113(61.1 \%)$ & \\
\hline MSAP & $120(23.5 \%)$ & 25 (19.1\%) & $43(22.2 \%)$ & $52(28.1 \%)$ & \\
\hline SAP & $34(6.7 \%)$ & $5(3.8 \%)$ & $9(4.6 \%)$ & $20(10.8 \%)$ & \\
\hline SIRS & $320(62.7 \%)$ & $32(24.4 \%)^{\#}$ & $63(32.5 \%)^{\&}$ & $95(51.4 \%)$ & 0.000 \\
\hline \multicolumn{6}{|l|}{ Scores of AP } \\
\hline Ranson Score & $1.2 \pm 1.2$ & $1.1 \pm 1.1^{\#}$ & $1.1 \pm 1.1^{\&}$ & $1.4 \pm 1.2$ & 0.032 \\
\hline CTSI Score & $3.3 \pm 2.0$ & $2.8 \pm 1.5^{\#}$ & $3.3 \pm 1.9$ & $3.6 \pm 2.2$ & 0.047 \\
\hline Organ failure & $154(30.2 \%)$ & $30(22.9 \%)^{\#}$ & $52(26.8 \%)^{\&}$ & 72 (38.9\%) & 0.004 \\
\hline \multicolumn{6}{|l|}{ Clinical indicators } \\
\hline WBC $\left(10^{9} / L\right)$ & $11.9 \pm 4.6$ & $10.5 \pm 4.2^{\#}$ & $11.8 \pm 4.7^{\&}$ & $12.9 \pm 4.5$ & 0.000 \\
\hline $\mathrm{GLU}(\mathrm{mmol} / \mathrm{L})$ & $8.8 \pm 4.0$ & $7.3 \pm 2.5^{\#}$ & $8.2 \pm 3.3^{\&}$ & $10.7 \pm 4.8$ & 0.000 \\
\hline $\mathrm{Cr}$ (umol/L) & $60.8(1.5-680)$ & $63.4(6.3-680){ }^{\#}$ & $60.8(1.5-430.0)$ & $57.9(17.0-338.0)$ & 0.017 \\
\hline BUN (mmol/L) & $4.8 \pm 2.3$ & $5.3 \pm 2.7$ & $4.6 \pm 2.1$ & $4.6 \pm 2.0$ & 0.162 \\
\hline LDH (IU/L) & 195.7(4.4-1461.0) & $200(4.4-786.0)$ & 200.5(18-860) & $192.0(22.8-1461.0)$ & 0.893 \\
\hline $\mathrm{TC}(\mathrm{mmol} / \mathrm{L})$ & $4.4(1.1-12.7)$ & $3.4(1.7-6.5)^{\#}$ & $4.4(1.6-12.7)^{8}$ & $5.6(1.1-12.7)$ & $<0.001$ \\
\hline TG $(\mathrm{mmol} / \mathrm{L})$ & $2.5(0.1-39.2)$ & $0.9(0.2-1.7)^{\#}$ & $2.4(0.1-39.2)^{\&}$ & $7.4(1.7-39.0)$ & $<0.001$ \\
\hline
\end{tabular}

All the shorthand is shown below: WBC white blood cells, $G L U$ glucose, $C r$ creatinine, $B U N$ blood urea nitrogen, $L D H$ lactate dehydrogenase, $T C$ total cholesterol, $T G$ triglyceride

arepresents comparing among the three groups;

\#The HTGW compared with the NWNT group, $P<0.05$

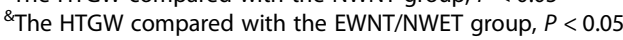

\section{Discussion}

Clinically, there are many possible causes of pancreatitis including biliary, alcohol, and hypertriglyceridemia, of which biliary factors are the most common [31-33]. In China, HTG-AP has replaced alcoholic AP as the second most common cause [23, 27]. Our results showed that $28.2 \%$ of patients exhibited biliary AP, $6.7 \%$ had alcoholic AP, and $40.2 \%$ of patients had HTG-AP, suggesting that hypertriglyceridemia is the most common cause of AP. When doctors judge patients as having biliary AP, they may pay more attention to possible biliary obstruction and fail to measure TG. Thus, these patients would have been excluded for having incomplete data in our study. In subgroup analysis, our data demonstrated that biliary factors were the main cause of AP (51.1\%) in the NWNT group. In contrast, the main cause of AP in the HTGW group was hypertriglyceridemia, which accounted for $67 \%$ of cases and met the definition of HTGW.

Obesity refers to an excessive deposition of adipose tissue. A meta-analysis of observational results showed that obesity (BMI $\left.>30 \mathrm{~kg} / \mathrm{m}^{2}\right)$ was a risk factor for SAP, local complications, SIRS, and increased rate of death [34]. Obesity was classified as abdominal or subcutaneous. Current clinical studies show that visceral fat, which exists in or around the pancreas, may worsen the prognosis of AP, whereas subcutaneous fat rarely affects prognosis [35]. Visceral fat is increasingly recognized as a risk factor for AP. Our data clearly showed that patients with SAP comprised $10.8 \%$ and patients with SIRS comprised $51.8 \%$ of the HTGW group, which was more severe than the composition of the other two groups. This finding is in agreement with earlier results [36, 37], suggesting that WC and hypertriglyceridemia have a clear correlation with AP severity.

It is difficult to measure visceral obesity as the measurements need to be verified using a specific software system [38]. However, such methods are more common in research, whereas their clinical use is limited. Lemieux first introduced the HTGW phenotype in the Quebec Cardiovascular Study and suggested that this phenotype may be an inexpensive tool for identifying asymptomatic patients at elevated risk of coronary heart disease [14]. Use of HTGW is based on the concept that abdominal fat and dyslipidemia are at the heart of the Mets pathogenesis. WC is a simple anthropometric value with good reproducibility, and the measurement of $\mathrm{WC}$ is convenient and inexpensive to obtain. However, WC does not distinguish between visceral and subcutaneous fat. Hypertriglyceridemia is a simple clinical marker of excessive visceral fat associated with increased WC. The HTGW phenotype, which indicates people with high WC and hypertriglyceridemia [20], 
Table 3 Correlation between disease severity and HTGW\%、WTI

\begin{tabular}{|c|c|c|c|c|c|}
\hline & Cohort $N=510$ & $n=356$ & MSAP $n=120$ & SAP $n=34$ & $P$ values ${ }^{a}$ \\
\hline Age years (mean $\pm S D)$ & $47.9 \pm 11.9$ & $47.7 \pm 11.8$ & $48.1 \pm 12.2$ & $48.7 \pm 11.8$ & 0.870 \\
\hline Male(\%) & $319(62.5 \%)$ & $225(63.2 \%)$ & $75(62.5 \%)$ & 19 (55.9\%) & 0.701 \\
\hline Smoking & $128(25.1 \%)$ & $82(23.0 \%)$ & $38(31.7 \%)$ & $8(23.5 \%)$ & 0.267 \\
\hline Drinking & $87(17.1 \%)$ & $60(16.9 \%)$ & $24(20.0 \%)$ & $3(8.8 \%)$ & 0.185 \\
\hline $\mathrm{BMI}^{*}$ & $25.5 \pm 5.1$ & $25.3 \pm 5.0$ & $25.8 \pm 5.6$ & $26.4 \pm 3.8$ & 0.449 \\
\hline Waist circumference & $87.0 \pm 9.4$ & $85.9 \pm 9.1^{\#}$ & $89.1 \pm 10.2$ & $90.6 \pm 8.1^{\$}$ & 0.000 \\
\hline WTI & $221.7(10.7-3712.6)$ & $209.5(15.1-3712.6)^{\#}$ & $229.5(20.2-3507.0)^{8}$ & $522.8(10.7-2804.0)^{\$}$ & $<0.001$ \\
\hline HTGW\% & 185 (36.3\%) & $113(31.7 \%)^{\#}$ & $52(43.3 \%)$ & $20(58.8 \%)$ & 0.001 \\
\hline Etiology & & & & & 0.075 \\
\hline Biliary & $144(28.2 \%)$ & $108(30.3 \%)$ & $28(23.3 \%)$ & $8(23.5 \%)$ & \\
\hline Alcohol & $34(6.7 \%)$ & $26(7.3 \%)$ & $7(5.8 \%)$ & $1(2.9 \%)$ & \\
\hline Hypertriglyceridemia & 205 (40.2\%) & $136(38.2 \%)$ & $48(40.0 \%)$ & $21(61.8 \%)$ & \\
\hline Others & 127 (24.9\%) & $86(24.2 \%)$ & 37 (30.8\%) & $4(11.4 \%)$ & \\
\hline WBC $\left(10^{9} / L\right)$ & $11.9 \pm 4.6$ & $11.1 \pm 4.1^{\#}$ & $13.6 \pm 5.1$ & $14.1 \pm 5.2^{\$}$ & 0.000 \\
\hline LDH (IU/L) & 195.7(4.4-1461.0) & 187.5(4.4-1461.0) & $222.0(110.0-786.0)$ & $313.0(139.0-1410.0)$ & 0.893 \\
\hline $\mathrm{GLU}(\mathrm{mmol} / \mathrm{L})$ & $8.8 \pm 4.0$ & $8.3 \pm 3.5^{\#}$ & $9.7 \pm 4.3^{\&}$ & $11.6 \pm 5.8^{\$}$ & 0.000 \\
\hline BUN (mmol/L) & $4.8 \pm 2.3$ & $4.5 \pm 1.9$ & $5.2 \pm 3.1$ & $5.8 \pm 2.0^{\$}$ & 0.039 \\
\hline $\mathrm{Cr}(\mathrm{mmol} / \mathrm{L})$ & $60.8(1.5-680)$ & $60.1(1.5-128.0)^{\#}$ & 63.3(17.0-181.0) & $69.5(30.0-680.0)$ & 0.017 \\
\hline $\mathrm{TG}(\mathrm{mmol} / \mathrm{L})$ & $2.5(0.1-39.2)$ & $2.4(0.2-39.0)$ & $2.5(0.2-39.2)^{\&}$ & $6.0(0.1-29.4)^{\$}$ & $<0.001$ \\
\hline TC $(\mathrm{mmol} / \mathrm{L})$ & $4.4(1.1-17.1)$ & $4.4(1.1-17.1)$ & $4.4(2.1-12.2)$ & $4.5(1.7-10.9)$ & 0.679 \\
\hline
\end{tabular}

All the shorthand are shown below: WBC white blood cells, $L D H$ lactate dehydrogenase, $G L U$ glucose,BUN blood urea nitrogen, $C r$ creatinine,and $T G$ triglyceride a represents comparing among the three groups;

" represents the MAP compared with the MSAP group, $P<0.05$

\& represents the MSAP compared with the SAP group, $P<0.05$

srepresents the MAP compared with the SAP group, $P<0.05$

is a simple and reliable phenotypic indicator of metabolic disease related to visceral obesity. This phenotype has been used as a screening indicator for cardiovascular disease, prodromal diabetes, and other diseases. So far, the correlation between the phenotype and AP has not been reported.

Our results indicated that patients with AP who exhibited the HTGW phenotype had a higher incidence of
SAP, SIRS, and organ failure. In addition, the phenotype was useful for predicting the occurrence of organ failure and SIRS. Multivariate logistic regression analysis showed that patients with the HTGW phenotype had 1.664 times the risk of organ failure and 1.891 times the risk of SIRS, compared with the other groups. Accordingly, the HTGW phenotype acts as a simple screening tool for early prediction of SAP.

Table 4 Logistic regression analysis of organ failure and SIRS in patients with AP

\begin{tabular}{|c|c|c|c|c|c|c|c|c|}
\hline \multicolumn{5}{|l|}{ Organ Failure } & \multicolumn{4}{|l|}{ SIRS } \\
\hline & B (S.E) & $P$ & OR & $95 \% \mathrm{Cl}$ & B (S.E) & $P$ & OR & $95 \% \mathrm{Cl}$ \\
\hline Male & 0.204 & 0.412 & 1.227 & $0.753-1.998$ & 0.250 & 0.299 & 1.283 & $0.801-2.056$ \\
\hline Age & 0.014 & 0.157 & 1.014 & 0.995-1.035 & 0.000 & 0.965 & 1.000 & $0.981-1.020$ \\
\hline BMI & 0.019 & 0.406 & 1.019 & $0.975-1.066$ & 0.051 & 0.035 & 1.052 & $1.004-1.103$ \\
\hline Hypertension & -0.383 & 0.411 & 0.682 & $0.273-1.701$ & 0.775 & 0.065 & 2.170 & $0.953-4.942$ \\
\hline Diabetes & 0.544 & 0.043 & 1.722 & $1.018-2.915$ & 0.614 & 0.024 & 1.848 & $1.083-3.153$ \\
\hline $\mathrm{CHD}$ & -0.034 & 0.961 & 0.966 & $0.242 \pm 3.860$ & -0.281 & 0.692 & 0.755 & $0.188-3.038$ \\
\hline Smoking & 0.486 & 0.083 & 1.626 & $0.938-2.818$ & 0.276 & 0.319 & 1.317 & $0.766-2.267$ \\
\hline Drinking & -0.191 & 0.548 & 0.826 & $0.442-1.543$ & -0.140 & 0.651 & 0.869 & $0.474-1.595$ \\
\hline HTGW & 0.509 & 0.036 & 1.664 & $1.033-2.680$ & 0.637 & 0.007 & 1.891 & $1.192-2.998$ \\
\hline
\end{tabular}

Abbreviations: WTI Waist circumference index,BMI Body Mass Index,95\% Cl confidence interval,OR odds ratio,CHD Coronary heart disease The left table represents the prediction of organ failure and the right represents the analysis of SIRS 
WTI, first described by R F Yang et al., is a quantitative indicator of the HTGW phenotype [15]. Our data showed that, as AP increased in severity, both the proportion of the HTGW phenotype and WTI significantly increased. It is worth noting that BMI, which only represents overall obesity, did not significantly differ among the MAP, MSAP, and SAP groups. This finding confirmed those of Sadr-Azodi; that is, abdominal obesity (rather than total obesity) was an independent risk factor for development of AP [36].

The HTGW phenotype may be closely related to AP severity via free fatty acids (FFAs). Both the lipolysis of adipose tissue and hydrolysis of triglyceride produce excessive FFAs after AP onset. Clinical research revealed that circulating FFAs are positively correlated with the severity of AP [39]. Furthermore, Substantial studies have shown that FFAs, especially unsaturated fatty acids (UFAs), exert damage on pancreatic acinar cells [40-42]. Patel et al. found that visceral fat triglyceride secreted excessive UFAs under the action of pancrelipase. This promoted the development of MAP into SAP, eventually triggered MODF and increased the mortality [41, 43]. Navina $S$ et al. also showed that UFAs, produced by necrotic peripancreatic visceral fat, aggravated pancreatic inflammation and promoted the transformation of MAP to SAP [42]. In addition, orlistat, a esterase inhibitor, could significantly attenuated the AP severity in obesity mice [41-43]. All the previous findings confirm the critical role of FFAs in the pathomechanism of AP patients with obesity.

There were several limitations to this study. Firstly, this was a retrospective analysis, and not all patients had their WC measured by soft ruler at admission. For these patients, WC could be only measured by abdominal CT. Abdominal CT is not an infallible measure of WC, which could have introduced some data bias. However, it still can get relatively accurate data, as described in Additional file 1 Figure S1. Obviously, we cannot directly compare WC obtained by CT with that obtained by soft ruler. Accordingly, the WC data of patients in this study were all obtained by abdominal CT in order to minimize error and avoid bias. Secondly, this was a single-center retrospective study with a small sample size. Multi-center studies are needed to validate our findings and further explore the clinical significance of the HTGW phenotype as an early indicator of SAP in patients with AP.

In summary, our results demonstrate that patients with AP, with the HTGW phenotype, have a higher risk of organ failure. WTI, as a quantitative indicator of the HTGW phenotype, was positively correlated with the severity of AP. The HTGW phenotype and WTI can be used as simple indicators for early identification of high-risk patients with SAP.

\section{Additional file}

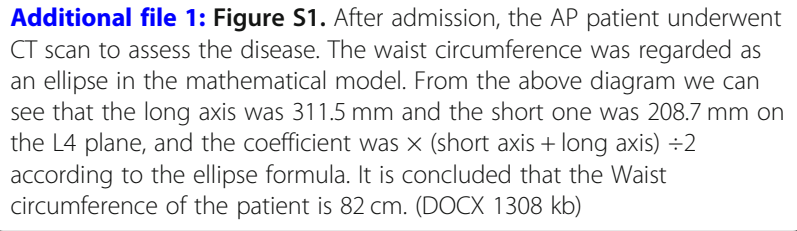

\section{Acknowledgements}

We appreciated the professional English Editor of Enago.

\section{Funding}

This study is supported by the National Natural Science Foundation of Yangzhou City (YZ2016128 and YZ2017106), the Postdoctoral Science Foundation of China (2018 M632260), and the Postdoctoral Science

Foundation of Jiangsu Province (1701047C).

\section{Availability of data and materials}

All data generated and analyzed during this study are included in this published article. The datasets are available from the corresponding author on reasonable request.

\section{Authors' contributions}

The study was designed by Guotao Lu, Yanbing Ding and Zhenglei Xu; Min Zhang, Lisheng Wang, Tao Yin, Ningzhi Wang, Jian Wu and Jiehua Zhi analyzed and interpreted the data; Weiwei Chen and Keyan Wu were responsible for drafting the manuscript; The manuscript was reviewed by Weijuan Gong and Weiming Xiao. All authors have read and approved the final manuscript for publication.

\section{Ethics approval and consent to participate}

Because of the retrospective nature of this study, informed consent was waived, and the study was approved by the Ethics Committee of our hospital.

\section{Consent for publication}

All authors provide consent for publication of this paper.

\section{Competing interests}

All authors of this paper have no conflicts of interests to disclose.

\section{Publisher's Note}

Springer Nature remains neutral with regard to jurisdictional claims in published maps and institutional affiliations.

\section{Author details}

1'Department of Gastroenterology, Affiliated Hospital of Yangzhou University, Yangzhou University, No. 386 Hanjiang Media Road, Yangzhou 225000, Jiangsu, China. 'Laboratory of Gastroenterology, Affiliated Hospital of Yangzhou University, Yangzhou University, No. 386 Hanjiang Media Road, Yangzhou 225000, Jiangsu, China. ${ }^{3}$ Department of Gastroenterology, The second Clinical Medical College (Shenzhen People's Hospital), Jinan University, Shenzhen 518000, Guangdong, China. ${ }^{4}$ Department of Gastroenterology, Clinical Medical College, Yangzhou University, Yangzhou, China. ${ }^{5}$ Department of Immunology, School of Medicine, Yangzhou

University, Yangzhou, China.

Received: 12 December 2018 Accepted: 14 March 2019

Published online: 09 April 2019

\section{References}

1. Lankisch PG, Apte M, Banks PA. Acute pancreatitis. Lancet. 2015;386:85-96

2. Garg SK, Sarvepalli S, Campbell JP, Obaitan I, Singh D, Bazerbachi F, et al. Incidence, admission rates, and predictors, and economic burden of adult emergency visits for acute pancreatitis: data from the National Emergency Department Sample, 2006 to 2012. J Clin Gastroenterol. 2019;53(3):220-5. 
3. PAVLIDIS PJ. Improved outcome of severe acute pancreatitis in the intensive care unit[J]. Crit Care Res Pract. 2013;2013:897107.

4. Forsmark CE,Vege SS,Wilcox CMN. Acute Pancreatitis. Engl J Med,2016,11,375,20,1972-1981.

5. Unamuno X, Gomez-Ambrosi J, Rodriguez A, Berrecil S, Fruhbeck G, Catalan $\checkmark$. Adipokine dysregulation and adipose tissue inflammation in human obesity. Eur J Clin Investig 2018:e12997.

6. Shin KY, Lee WS, Chung DW, Heo J, Jung MK, Tak WY, et al. Influence of obesity on the severity and clinical outcome of acute pancreatitis. Gut and liver. 2011:5:335-9.

7. Khatua B, El-Kurdi B, Singh VP. Obesity and pancreatitis. Curr Opin Gastroenterol. 2017;33:374-82.

8. Chen SM, Xiong GS, Wu SM. Is obesity an indicator of complications and mortality in acute pancreatitis? An updated meta-analysis. J Digest Dis. 2012;13:244-51.

9. Natu A, Stevens T, Kang L, Yasinow S, Mansoor E, Lopez R, et al. Visceral adiposity predicts severity of acute pancreatitis. Pancreas. 2017:46:776-81.

10. Garg R, Rustagi T. Management of Hypertriglyceridemia Induced Acute Pancreatitis. Biomed Res Int. 2018;2018:4721357.

11. Adiamah A, Psaltis E, Crook M, Lobo DN. A systematic review of the epidemiology, pathophysiology and current management of hyperlipidaemic pancreatitis.Clin Nutr. 2018;37(6 Pt A):1810-1822.

12. Wang Q, Wang G, Qiu Z, He X, Liu C, et al. Elevated serum triglycerides in the prognostic assessment of acute pancreatitis: a systematic review and metaanalysis of observational studies. J Clin Gastroenterol. 2017;51(7):586-93.

13. Nawaz H, Koutroumpakis E, Easler J. Elevated serum triglycerides are independently associated with persistent organ failure in acute pancreatitis. Am J Gastroenterol. 2015;110(10):1497-503.

14. Lemieux I, Pascot A, Couillard C, Lamarche B, Tchernof A, Alméras N, et al. Hypertriglyceridemic waist : a marker of the Atherogenic metabolic triad (hyperinsulinemia; Hyperapolipoprotein B; small, dense LDL) in men? Circulation. 2000;102:179-84.

15. Yang RF, Liu XY, Lin Z, Zhang G. Correlation study on waist circumferencetriglyceride (WT) index and coronary artery scores in patients with coronary heart disease. Eur Rev Med Pharmacol Sci. 2015;19:113-8.

16. He S, Zheng Y, Shu Y, He J, Wang Y, Chen X. Hypertriglyceridemic waist might be an alternative to metabolic syndrome for predicting future diabetes mellitus. PLoS One. 2013;8:e73292.

17. Chen S, Guo X, Yu S, Yang H, Sun G, Li Z, et al. Hypertriglyceridemic waist phenotype and metabolic abnormalities in hypertensive adults: a STROBE compliant study. Medicine. 2016;95:e5613.

18. Vaverkova H, Karasek D, Novotny D, Halenka M, Orsag J, Slavik L. Hypertriglyceridemic waist - a simple clinical tool to detect cardiometabolic risk: comparison with harmonized definition of metabolic syndrome. Physiol Res. 2015;64(Suppl 3):S385-94.

19. Wang A, Li Z, Zhou Y, Wang C, Luo Y, Liu X, et al. Hypertriglyceridemic waist phenotype and risk of cardiovascular diseases in China: results from the Kailuan study. Int J Cardiol. 2014;174:106-9.

20. Zhang M, Gao Y, Chang H, Wang X, Liu D, Zhu Z, et al. Hypertriglyceridemic-waist phenotype predicts diabetes: a cohort study in Chinese urban adults. BMC Public Health. 2012;12:1081.

21. Janghorbani M, Salamat MR, Aminorroaya A, Amini M. Utility of the Visceral Adiposity Index and Hypertriglyceridemic Waist Phenotype for Predicting Incident Hypertension. Endocrinol Metab (Seoul). 2017. 32: 221-229.

22. Wu BU, Batech M, Quezada M, Lew D, Fujikawa K, Kung J, et al. Dynamic measurement of disease activity in acute pancreatitis: the pancreatitis activity scoring system. Am J Gastroenterol. 2017;112:1144-52.

23. Zhu Y, Pan $X$, Zeng $H$, He W, Xia L, Liu P, et al. A study on the etiology, severity, and mortality of 3260 patients with acute pancreatitis according to the revised Atlanta classification in Jiangxi, China over an 8-year period. Pancreas. 2017;46(4):504-9.

24. van Geenen EJM, van der Peet DL, Bhagirath P, Mulder CJ, Bruno MJ, et al. Etiology and diagnosis of acute biliary pancreatitis. Nat Rev Gastroenterol Hepatol. 2010;7(9):495-502.

25. de Pretis N, Amodio A, Frulloni L. Hypertriglyceridemic pancreatitis: epidemiology, pathophysiology and clinical management. United European Gastroenterol J. 2018;6(5):649-55.

26. Scherer J, Singh VP, Pitchumoni CS, Yadav D. Issues in hypertriglyceridemic pancreatitis: an update. J Clin Gastroenterol. 2014;48(3):195-203.

27. Yin G, Cang X, Yu G, Hu G, Ni J, Xiong J, et al. Different clinical presentations of Hyperlipidemic acute pancreatitis: a retrospective study. Pancreas. 2015; 44(7):1105-10.
28. Banks PA, Bollen TL, Dervenis C, Gooszen HG, Johnson CD, Sarr MG, et al. Classification of acute pancreatitis--2012: revision of the Atlanta classification and definitions by international consensus. Gut. 2013;62:102-11.

29. Goyal H, Guerreso K, Smith B, Harper K, Patel S, Patel A, et al. Severity and outcomes of acute alcoholic pancreatitis in cannabis users. Transl Gastroenterol Hepatol. 2017;2:60.

30. Cho JH, Kim TN, Kim SB. Comparison of clinical course and outcome of acute pancreatitis according to the two main etiologies: alcohol and gallstone. BMC Gastroenterol. 2015 Jul 25;15:87. https:/doi.org/10.1186/ s12876-015-0323-1.

31. Fan J, Ding L, Lu Y, Zheng J, Zeng Y, Huang C. Epidemiology and etiology of acute pancreatitis in urban and suburban areas in Shanghai: a retrospective study. Gastroenterol Res Pract. 2018;2018:1420590.

32. Sakorafas GH, Tsiotou AG. Etiology and pathogenesis of acute pancreatitis: current concepts. J Clin Gastroenterol. 2000;30:343-56.

33. Gao YJ, Li YQ, Wang Q, Li SL, Li GQ, Ma J, et al. Analysis of clinical features of acute pancreatitis in Shandong Province, China. J Gastroenterol Hepatol. 2007:22:340-4

34. Martinez J, Johnson CD, Sanchez-Paya J, de Madaria E, Robles-Diaz G, PerezMateo M. Obesity is a definitive risk factor of severity and mortality in acute pancreatitis: an updated meta-analysis. Pancreatology : official journal of the International Association of Pancreatology. 2006;6:206-9.

35. Abu Hilal M, Armstrong T. The impact of obesity on the course and outcome of acute pancreatitis. Obes Surg. 2008;18:326-8.

36. Sadr-Azodi O, Orsini N, Andren-Sandberg A, Wolk A. Abdominal and total adiposity and the risk of acute pancreatitis: a population-based prospective cohort study. Am J Gastroenterol. 2013;108:133-9.

37. Nawaz H, Koutroumpakis E, Easler J, Slivka A, Whitcomb DC, Singh VP, et al. Elevated serum triglycerides are independently associated with persistent organ failure in acute pancreatitis. Am J Gastroenterol. 2015;1 10(10):1497-503.

38. Makino $\mathrm{H}$, Kunisaki $\mathrm{C}$, Akiyama $\mathrm{H}$, et al. Effect of obesity on intraoperative bleeding volume in open gastrectomy with D2 lymph-node dissection for gastric cancer. Patient safety in surgery. 2008;2:7.

39. Sztefko K, Panek J. Serum free fatty acid concentration in patients with acute pancreatitis. Pancreatology. 2001;1(3):230-6.

40. Chang YT, Chang MC, Tung CC, Wei SC, Wong JM, et al. Distinctive roles of unsaturated and saturated fatty acids in hyperlipidemic pancreatitis. World J Gastroenterol. 2015;21(32):9534-43.

41. Noel P, Patel K, Durgampudi C, Trivedi RN, Trivedi RN, de Oliveira C, Crowell $M D$, et al. Peripancreatic fat necrosis worsens acute pancreatitis independent of pancreatic necrosis via unsaturated fatty acids increased in human pancreatic necrosis collections. Gut. 2016;65(1):100-11.

42. Navina S, Acharya C, DeLany JP, Orlichenko LS, Baty CJ, Shiva SS, et al. Lipotoxicity causes multisystem organ failure and exacerbates acute pancreatitis in obesity. Sci Transl Med. 2011 3(107):107ra110.

43. Patel K, Trivedi RN, Durgampudi C, Noel P, Cline RA, DeLany JP, et al. Lipolysis of visceral adipocyte triglyceride by pancreatic lipases converts mild acute pancreatitis to severe pancreatitis independent of necrosis and inflammation. Am J Pathol. 2015;185(3):808-19.

\section{Ready to submit your research? Choose BMC and benefit from:}

- fast, convenient online submission

- thorough peer review by experienced researchers in your field

- rapid publication on acceptance

- support for research data, including large and complex data types

- gold Open Access which fosters wider collaboration and increased citations

- maximum visibility for your research: over 100M website views per year

At $\mathrm{BMC}$, research is always in progress.

Learn more biomedcentral.com/submissions 